\title{
Supercontinuum generation from intense partially coherent laser beams in optical fibers
}

\author{
P. Corredera ${ }^{(1)}$, S. Martín-López ${ }^{(1)}$, and M. González-Herráez ${ }^{(2)}$ \\ ${ }^{(1)}$ Departamento de Metrología, Instituto de Física Aplicada CSIC, Madrid, Spain. \\ ${ }^{(2)}$ Departamento de Electrónica, Universidad de Alcalá, Madrid, Spain.
}

\begin{abstract}
This paper analyses the advantageous features of supercontinuum (SC) generated from continuous-wave (CW) excitation. It has been shown that both the generation mechanisms and the temporal and spectral properties of supercontinuum produced with $\mathrm{CW}$ pump lasers are different from those of generated by means of pulsed excitation, in particular the remarkable smoothness of CW-SC spectra. We show that these unique spectral features stem from the fission of the partially coherent $\mathrm{CW}$ input beam into a train of subpicosecond pulses induced by the modulation instability (MI). These subpicosecond pulses lead to the formation of optical solitons with inherently random parameters, which self-frequency shift differently depending on their characteristics. The resulting supercontinuum spectrum is hence the average of many different soliton spectra, which have suffered different frequency shifts. Different experimental setups used in our lab are presented and the dependence of the SC generation on the coherence of the fiber laser and the fiber dispersion profile are shown.
\end{abstract}

Keywords: supercontinuum, modulation instability, nonlinear optics.

\section{INTRODUCTION}

Nonlinear spectral broadening and supercontinuum (SC) generation in optical fibers has been the subject of many studies in the past few years ${ }^{1-4}$. To date, most of the experiments have been performed using high-peak-power nanosecond, picosecond, or femtosecond pulses and/or special fibers such as photonic crystal fibers or dispersion-tapered fibers. CWSC generation in optical fibers has attracted much attention in the past few years for the possibility of developing compact, high-quality sources for ultrahigh resolution optical coherence tomography $(\mathrm{OCT})^{5-7}$. Among their good properties, these sources exhibit extremely low coherence lengths (allowing resolutions of several micrometers in OCT applications), high power spectral densities (normally of the order of several $\mathrm{mW} / \mathrm{nm}$ ), and lower values of relative intensity noise than their pulsed counterparts. More recently, nonlinear pump spectral broadening of $\mathrm{CW}$ beams has been demonstrated as an effective tool to develop spectrally flattened Raman amplifiers ${ }^{9-11}$.

One of the basic problems in the study of supercontinuum generation consists in searching the optimal regime of spectral broadening from the point of view of the target application (metrology, tomography, spectroscopy, and communications). The complexity of this problem is related to the fact that the contributions of various nonlinear-optical effects to the spectral broadening can be significantly different depending on the dispersion shape of the optical fiber and the exact temporal characteristics of the pumping radiation. In particular, the generation mechanisms and some of the SC properties can differ substantially for systems with $\mathrm{CW}$ and pulsed excitation. In addition, different applications impose different and, sometimes, opposite requirements on SC radiation, that makes impossible to create an all-purpose scheme for SC generation, which meets the requirements for all of the applications. For example, high-resolution optical coherent tomography (OCT) imaging of biological tissues requires a broadband $\mathrm{SC}^{12}$ with low relative intensity noise (RIN). In contrast, the supercontinua used for telecommunications ${ }^{9-11}$ based on wavelength-division multiplexing (WDM) can be relatively narrow to span the conventional telecommunications spectral range (1500-1600 nm), but the uniformity of the SC spectral power is important to eliminate the need for additional equalizing spectral filters. For timeresolved spectroscopy, a flat SC spectrum and a single-pulse distribution of the SC intensity in the time domain are also required. Each of these characteristics can be obtained by a suitable choice of the laser and the dispersive characteristics 
of the fiber used. For instance, single pulses are only generated when the self-phase modulation serves as the main mechanism for the SC generation (pulsed laser source and normally dispersive fiber). In this case, the spectral width is relatively small (only several tens of terahertz). Much larger spectral broadening can be realized when the SC generation is done in the anomalous dispersion regime of the fiber. However, the corresponding distribution of the SC intensity in the time domain is extremely complicated and corresponds to multiple pulses.

Completely different requirements to SC radiation are imposed, for instance, when the target application is their

use in counter-propagating pumping schemes for broadband fiber SRS amplifiers used in telecommunications ${ }^{10-11-13}$. In this case the time distribution of the SC intensity is relatively unimportant and the spectral power and its wavelength dependence play the key role. The problem of the SC intensity profile with respect to the radiation wavelength also emerges in metrology in different ways: for the development of optical clocks, the SC spectral power profile must cover at least one octave with no special requirements on shape. However, in the case of optical detector calibration, the optical SC must be spectrally wide, relatively flat, and the noise must be as low as possible ${ }^{13}$.

In this paper we aim to explain the dynamics of the process so as to understand what are the specific solutions adopted for each application. In particular, we will address continuous-wave SC sources and we will explain the remarkable smoothness of its spectrum. We show that these unique spectral features stem from the fission of the quasi$\mathrm{CW}$ input beam into a train of subpicosecond pulses induced by the modulation instability. These subpicosecond pulses lead to the formation of optical solitons with inherently random parameters, which self-frequency shift differently depending on their characteristics. The resulting supercontinuum spectrum is hence the average of many different soliton spectra, which have suffered different frequency shifts. Experimental results of CW-SC generated in our laboratory will be presented and in particular the study of the dependence of SC generation on the pump laser coherence and fiber dispersion profile will be shown.

\section{SUPERCONTINUUM GENERATION UNDER CW EXCITATION}

Light radiation propagating through a nonlinear medium experiences spectral broadening under certain conditions. The starting point to describe the non-linear propagation of an optical field in the fiber is the generalized nonlinear Schrödinger equation for a scalar electric field envelope $\mathrm{E}(\mathrm{z}, \mathrm{t})^{14}$ :

$$
\frac{d E}{d z}=-\frac{\alpha}{2} E-\beta_{1} \frac{\partial E}{\partial t}-\frac{i \beta_{2}}{2} \frac{\partial^{2} E}{\partial t^{2}}+\frac{\beta_{3}}{6} \frac{\partial^{3} E}{\partial t^{3}}+i \gamma\left(1+\frac{i}{\omega_{0}} \frac{\partial}{\partial t}\right)\left(E(z, t) \int_{-\infty}^{+\infty} R\left(t^{\prime}\right)\left|E\left(z, t-t^{\prime}\right)\right|^{2} d t^{\prime}\right)
$$

where $\mathrm{z}$ is the longitudinal fiber coordinate and $\mathrm{t}$ is the retarded time. The first term on the right is the losses in the fiber, the terms affected by $\beta_{i}$ give rise to all the dispersive effects happening in the fiber and the terms affected by $\gamma$ (fiber nonlinear coefficient) collect all the non linear effects happening in the fiber, all of them due to the third-order susceptibility of silica (Kerr effects, four wave mixing, Raman effects...).

A nice description of the different non linear effects and their influence in the spectral broadening can be found in the review done by Dudley et al. ${ }^{15}$. As a short summary, in the case of SC generated in the femtosecond regime, Raman scattering and self-phase modulation (SPM) are the dominant effects in the broadening processes for the case of normal GVD (Group Velocity Dispersion) pumping; when the pump is in the anomalous GVD regime, spectral broadening arises from soliton propagation: soliton fission and related dynamics. In supercontinua generated by longer pulses (from picoseconds to the CW regime), SPM and Raman are the dominating mechanics in the normal GVD regime; and, in the anomalous GVD regime modulation instability (MI), four wave mixing (FWM), Raman scattering and Raman self-frequency shift (RSFS) are the dominating mechanisms.

Thus, the main significant difference between the mechanisms of spectral broadening of CW-SC from the SC generation under short pulsed pumping is related to the non-linear effect of modulation instability. Indeed MI causes the noise amplification at the initial stage of the CW-radiation propagation and the decay of this radiation into a series of pulses. After the pulses have been formed, the spectral broadening occurs due to the same mechanisms as in the case of pulsed pumping. Centering our attention in the SC generated in standard telecommunication fibers, the spectral broadening and soliton formation in anomalous dispersion regime with psec-pulse pumping is initiated by SPM which induces an initial pulse compression followed by soliton formation ${ }^{16-17}$. In the case of a CW pump, MI is the process that 
leads to a symmetry breaking of the partially coherent input beam. The frequency-dependent gain coefficient of the MI, under the assumption of a coherent pump is given by the expression:

$$
g(\Omega)=\left|\beta_{2} \Omega\right|\left(\Omega_{C}^{2}-\Omega^{2}\right)
$$

with

$$
\Omega_{C}=\sqrt{\frac{4 \gamma P_{I N} e^{-\alpha z}}{\beta_{2}}}
$$

where $\gamma$ is the fiber nonlinear coefficient, $\beta_{2}$ is the group velocity dispersion coefficient, $\alpha$ is the fiber loss coefficient, and $P_{I N}$ is the input power. The MI causes power transfer from the CW pump to the intensity noise and generates two spectral bands symmetrically positioned at both sides of the initial pump in the spectrum, with a maximum at

$$
\Omega_{M A X}= \pm \sqrt{\frac{2 \gamma P_{I N} e^{-\alpha z}}{\beta_{2}}}
$$

In the time domain, the CW beam is converted into a periodic pulse train with the period $\mathrm{T}=2 \pi / \Omega_{\mathrm{MAX}}$. The separation of the side peaks depends on the characteristics of the fiber. Fig 1(a) shows the theoretical increasing gain spectral width and amplitude with increasing input power, for a dispersion shifted fiber (DSF) with $\beta_{2}=0.56 \mathrm{ps}^{2} / \mathrm{km}$ and $\gamma$ $=1.7 \mathrm{~W}^{-1} \mathrm{~km}^{-1}$. Fig 1 (b) shows the decreasing gain bandwidth for increasing absolute values of $\beta_{2}$. A low dispersion leads to a more substantial broadening. Therefore, the dependence of MI characteristics on input power, dispersion and nonlinear coefficient can be used to select the best combination of pump/fiber parameters in order to achieve optimal pump broadening at any given frequency.

(a)

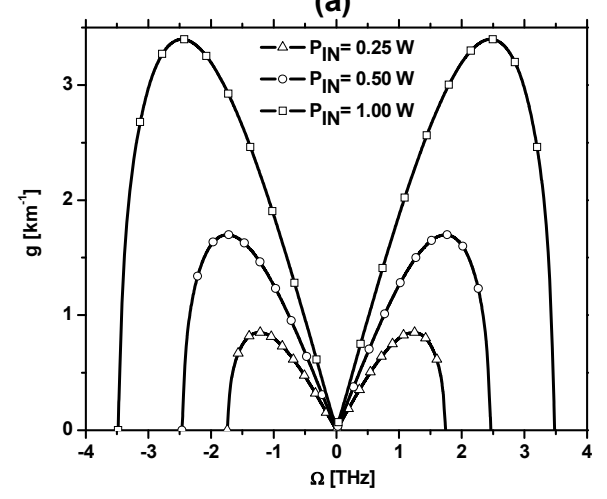

(b)

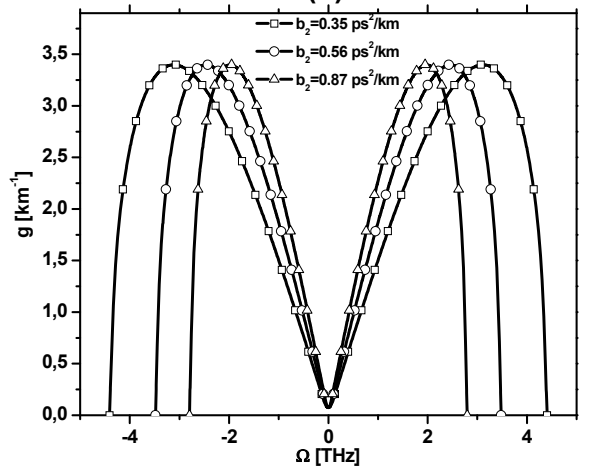

Fig 1: (a) Theoretical MI gain spectrum $(\mathrm{g}(\Omega))$ calculated for a DSF, $\left(\beta_{2}=0.56 \mathrm{ps}^{2} / \mathrm{km}\right.$ and $\left.\gamma=1.7 \mathrm{~W}^{-1} \mathrm{~km}^{1}\right)$ for three different input powers. (b) Theoretical MI gain spectrum calculated for different dispersion values and input power $\mathrm{P}_{\mathrm{IN}}=0.5 \mathrm{~W}$.

In Fig 2 the experimental MI spectra measured at our laboratory are depicted. Fig 2 (a) shows the spectra obtained for two different input powers: $\mathrm{P}_{1}=200 \mathrm{~mW}$ and $\mathrm{P}_{2}=250 \mathrm{~mW}$. Fig 2 (b) shows the output spectra obtained for the same input power $P_{1}=200 \mathrm{~mW}$ and for different pump wavelengths: $1556 \mathrm{~nm}, 1558 \mathrm{~nm}$ and $1561 \mathrm{~nm}$. The theoretical expected values of the frequency shifts are $\sim 0.22 \mathrm{THz}, \sim 0.18 \mathrm{THz}$ and $\sim 0.14 \mathrm{THz}$ for each of the pump wavelengths respectively. Once CW beam has broken into a series of pulses, if the energy of these pulses is sufficiently high they can evolve into solitons. These solitons propagate along the fiber in the absence of dispersion broadening with a red-shifting spectrum due to the SRS (Raman self-frequency shift, RSFS). Additionally, as they red-shift, they lose part of their energy, that is simultaneously transferred to the short-wavelength spectral range. This process is known as dispersivewave generation. 
(a)

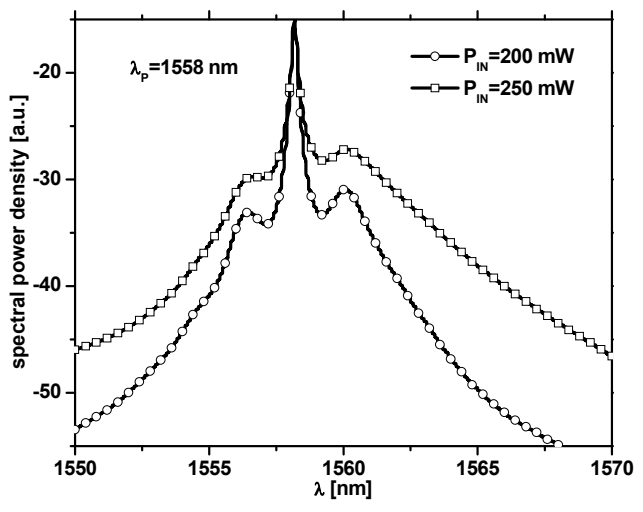

(b)

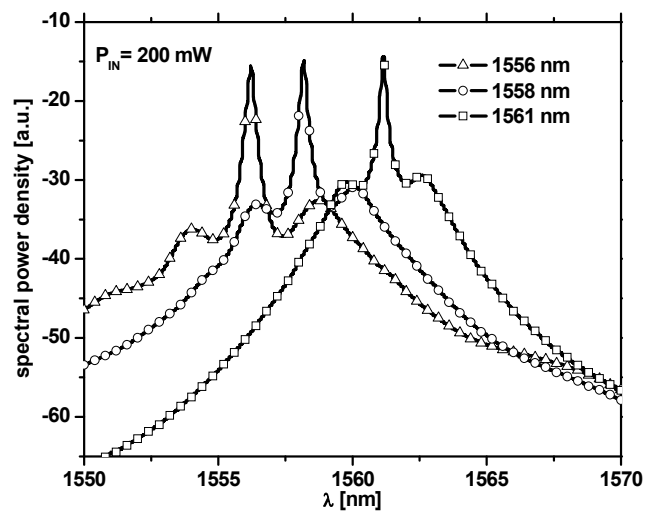

Fig 2. (a) Experimental MI spectra produced with a CW tunable laser at different wavelengths in a DSF with $\beta_{2}=0.56 \mathrm{ps}^{2} / \mathrm{km}$ at $1558 \mathrm{~nm}$ and $\gamma=1.7 \mathrm{~W}^{-1} \mathrm{~km}^{1}$ for $\mathrm{P}_{\mathrm{IN}}=0.2$ and $0,25 \mathrm{~W}$. (b) Experimental MI spectra produced using the same laser pumping and the same optical fiber, at different wavelengths with an input power $\mathrm{PIN}=0.2 \mathrm{~W}$.

\section{THE ROLE OF PUMP INCOHERENCE IN CW-SC GENERATION}

MI causes a substantial difference between CW- and pulse-pumped SC. Since noise amplification plays a key role at the initial stage of SC generation under CW pumping the temporal structure of such super-continuum is non-periodical. It contains a sequence of pulses with different wavelength, energy and duration and separated with random time intervals. Such irregular time structure makes CW-SC unsuitable for some applications such as telecom, optical frequency metrology, time-resolved spectroscopy and others. In general, SC generation under $\mathrm{CW}$ pumping can only be interesting for applications that are insensitive to fluctuations in the time distribution of the intensity: OCT, confocal microscopy, SRS fiber amplifiers with backward pumping, spectroscopy in the absence of time resolution and, possibly, several other applications.

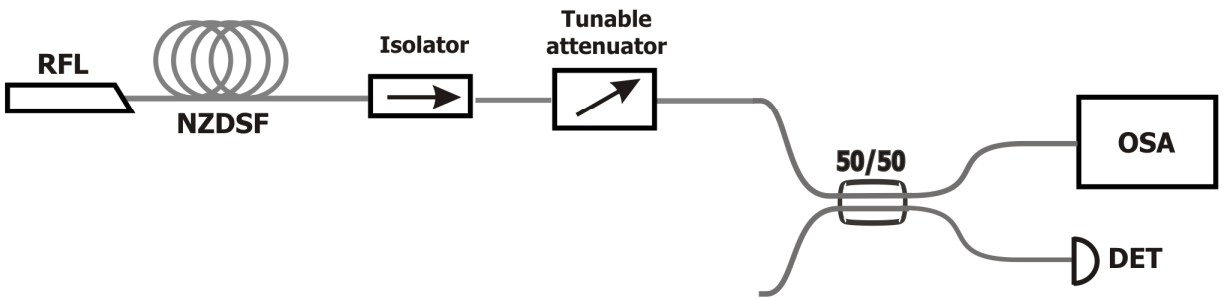

Fig 3: RFL: Raman Fiber Laser, NZDSF: non zero dispersion shift fiber Lucent TrueWave-RS ${ }, \mathrm{DET}$ : integrating sphere radiometer, OSA optical spectrum analyzer.

The incoherence of the $\mathrm{CW}$ source plays the role of the pump laser noise that, in the pulsed case, is known to seed the initial spectral broadening. Moreover, the random character of the fluctuations present in the incoherent pump beam explains the smoothness of the CW-SC spectra. In order to get a deeper understanding of these smooth spectra some simple experiments were make in our laboratory ${ }^{16-18}$. Fig 3 shows the experimental setup. A Raman fiber laser (RFL) emitting at $1455.3 \mathrm{~nm}$ with a maximal power of $2.1 \mathrm{~W}$ and FWHM at the output laser of $1.1 \mathrm{~nm}$ is used as SC pump source. This laser is launched to a $6.8 \mathrm{~km}$ length nonzero dispersion-shifted fiber (NZDSF) with $\gamma=2.7 \mathrm{~W}^{-1}$ $\mathrm{km}^{-1}, \beta_{2}=-0.11 \mathrm{ps}^{2} / \mathrm{km}, \beta_{3}=0.06 \mathrm{ps}^{3} / \mathrm{km}$ and zero dispersion wavelength at $1453 \mathrm{~nm}$, assuring a small anomalous dispersion $\left(\beta_{2}<0\right)$ at the pump wavelength in order to enhanced phase-matched FWM processes. The generated CW-SC spectrum has been measured for different input power using an optical spectrum analyzer (OSA). Fig 4 (a) shows the spectra of the laser pump for the different pump power levels and Fig 4 (b) the SC spectra obtained at the fiber end. For low pump power levels $\left(\mathrm{P}_{\mathrm{p}} \sim 0.3 \mathrm{~W}\right)$, the output spectrum exhibits MI sidebands around the pump frequency. The asymmetry in the intensity of those sidebands is caused by SRS amplification of the longer wavelengths and attenuation 
of the shorter ones. For higher pump powers, the effective FWM and Raman gains increase, which leads to a continuous broadening of the generated spectrum. At pump power $\mathrm{P}_{\mathrm{p}} \sim 1.2 \mathrm{~W}$, all the pump energy has been transferred to the SC, and at this point, the SC has a $20-\mathrm{dB}$ bandwidth of over $200 \mathrm{~nm}$. This SC spectrum exhibits a very smooth spectral structure at all pump power levels and that it broadens very regularly with increasing pump powers.

(a)

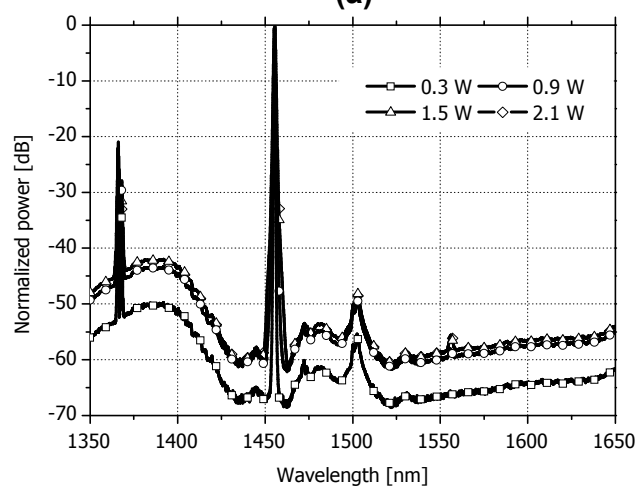

(b)

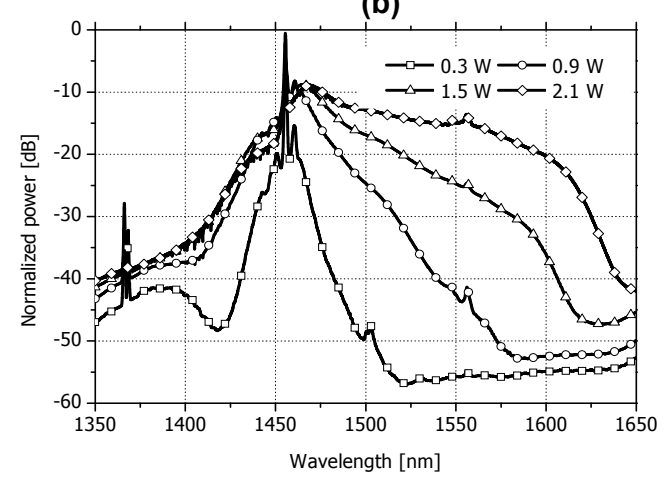

Fig 4. (a) Spectra of the laser pump for the different pump powers $\left(\mathrm{P}_{\mathrm{p}}\right)$. (b) SC spectrum obtained at the fiber end for the $\mathrm{P}_{\mathrm{p}}$ shown in (a).
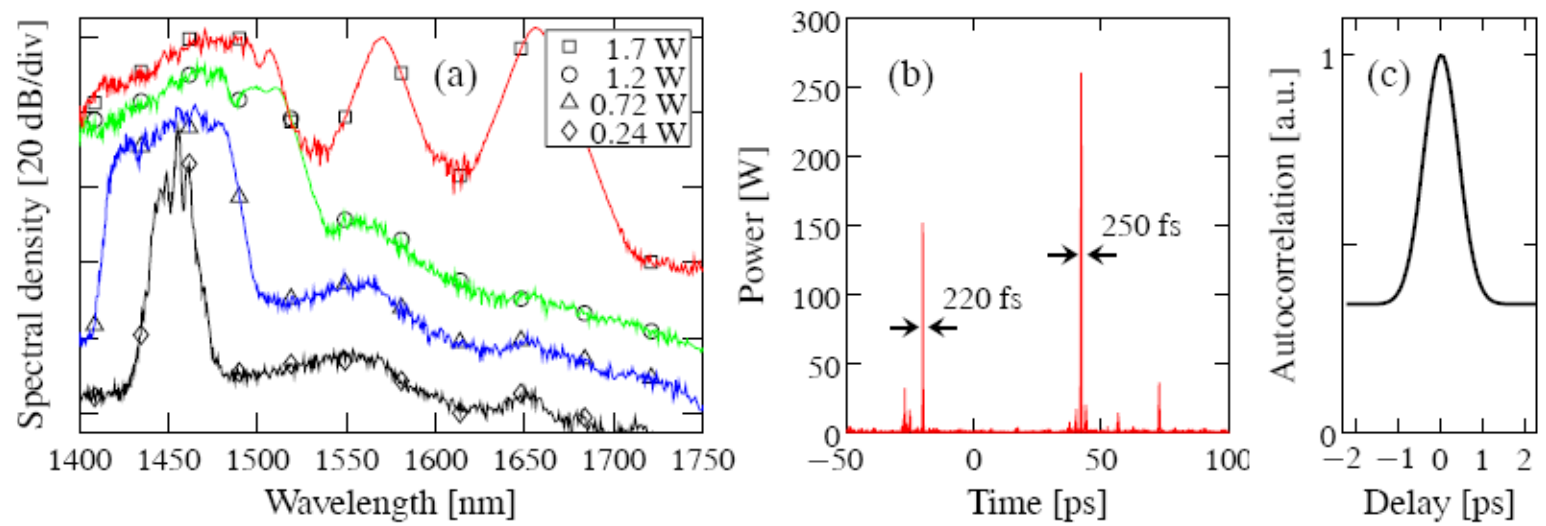

Fig 5. (a) Numerical output spectra for various pump power levels and (b) temporal intensity output at $\mathrm{Pp}=1.7 \mathrm{~W}$ for the initial condition shown in Fig. 2(a). (c) Measured intensity autocorrelation of the SC at Pp $=1.7 \mathrm{~W}$.

In order to understand the smooth observed spectra of the CW-SC in contrast with the SC generated by pico- or femtosecond pulsed pump, a numerical model based on generalized nonlinear Schrödinger equation (GNLSE) and numerically integrated by a split-step Fourier algorithm is used. Particular attention has been put to the initial condition, which in our case is supposed to represent the light emitted by a CW cascaded RFL. In practice, real CW lasers are only partially coherent and present a non vanishing spectral width. This means that these lasers exhibit random phase and/or intensity fluctuations on time scales of the order of their coherence time. Earlier works have shown that such partially coherent $\mathrm{CW}$ beams can undergo a significant amount of spectral broadening through, e.g, $\mathrm{SPM}^{19-20}$ and this contribution has to be taken into account in our CW-SC generation simulations. A more detailed explanation of the numerical simulations are $\mathrm{in}^{17}$. The output spectra corresponding to an arbitrary temporal intensity laser profile after propagation in our NZDSF for various pump power levels has been simulated. The results are presented in Fig 5(a). This figure shows that the low power simulations $(0.72 \mathrm{~W})$ agree fairly well with the experimental results (Fig $4(\mathrm{~b}))$ as they reproduce the MI sideband growth and the pump broadening. However, higher pump power simulations depart significantly from the experiments. In particular, the supercontinuum smoothness is not reproduced at all and we can clearly see, for the highest pump power, the generation of soliton-like features red-shifted from the pump beam. The solitonic nature of these spectral features can be readily confirmed by observing the corresponding temporal intensity output (Fig 5(b)). In this 
case, two ultrashort pulses of 220 fs and 250 fs (FWHM) can be clearly seen. While the presence of these fs solitons might seem surprising when considering that we are in principle dealing with a $\mathrm{CW}$ laser output, these are not mere numerical artifacts as can be shown by observing the experimental intensity autocorrelation trace of the SC output at 1.7 W pump power (Fig 5(c)). The observed pulses are actually Raman solitons whose generation is seeded from the modulation instability (MI)-induced break-up of the temporal fluctuations present in the initial condition. As these solitons propagate down the fiber, they undergo a continuous shift to longer wavelengths because of the Raman selffrequency shift.

The real situation of the CW-SC spectra measured in our laboratory is that the measures are the average of many different situations for random temporal intensity profiles of the similar laser output (these are integrated by the sweep time of the spectrum analyzer). To verify this interpretation, we have performed such an ensemble average numerically over 100 identical simulations. The result of these calculations is plotted in Fig 6 (solid curves) for 0.72 and $1.7 \mathrm{~W}$ pump power and is compared to the corresponding experimental spectra (dashed curves). As can be seen, the agreement is excellent. The SC smoothness is now reproduced correctly.
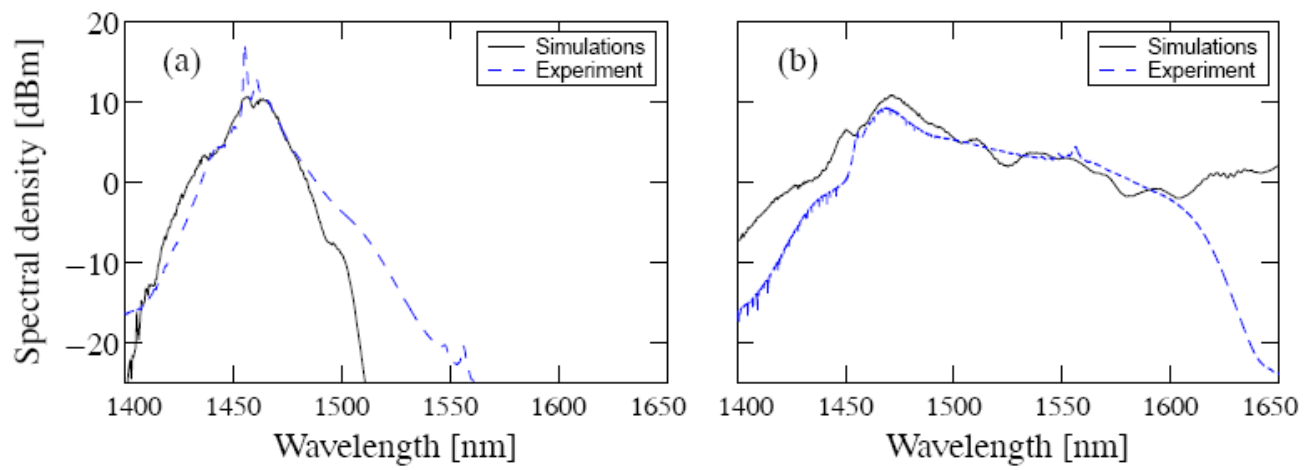

Fig 6. Comparison of experimental and simulated SC spectra obtained for (a) $\mathrm{P}_{\mathrm{p}}=0.72 \mathrm{~W}$ and (b) $\mathrm{P}_{\mathrm{p}}=1.7 \mathrm{~W}$.

\section{INFLUENCE OF CHROMATIC DISPERSION}

Chromatic dispersion plays a key role in the process of modulation instability and soliton formation. The most obvious limit is that these processes efficiently occur when pumping in the anomalous dispersion region of the fiber. Besides, at smaller anomalous dispersion values the MI oscillations are temporally faster and can induce shorter pulse formation. This effect leads to the generation of shorter solitons which suffer a larger soliton self-frequency shift (SSFS) per fiber length due to Raman scattering. Chromatic dispersion can also be engineered so as to induce soliton compression by the use of proper dispersion management, leading to enhanced supercontinuum spectra. An exhaustive experimental study of the influence of chromatic dispersion on CW-SC broadening in a standard DSF was realized in our laboratory ${ }^{21}$.

This study was done by tuning the wavelength of the high-power pump laser over the spectral region of small and moderate anomalous chromatic dispersion. Fig 7(a) shows the setup realized, based in a wavelength-tunable Erbium doped fiber ring laser (7W output power and a spectral width of $0.4 \mathrm{~nm}$ ). Fig 7(b) shows the output SC spectra for input power of $1 \mathrm{~W}$. It is clear in this figure the apparition of dispersive waves (DW). These DW arrive from the fact of the MI-generated pulses evolve into fundamental first-order solitons, which undergo a spectral shift towards longer wavelengths due to SRS. Each fundamental soliton can, in the presence of higher-order dispersion, release excess energy that emerging as the dispersive wave, enhancing the spectral broadening in the normal dispersion region of the fiber. These DW are emitted only when the soliton spectrum overlaps with some resonant wave frequencies. The amplitude of the DW radiation is proportional to the amplitude of the soliton at the phase-matched frequencies, so that more intense radiation is released by solitons that are closer to $\lambda_{\mathrm{ZD}}$. Returning to Fig $7(\mathrm{~b})$, at $1 \mathrm{~W}$ input power, MI sidebands cannot be appreciated and large spectral broadening and strong pump depletion start to take place. For a large detuning between the pump and $\lambda_{\mathrm{ZD}}$ (pumping at $1570 \mathrm{~nm}$ ), the soliton spectrum does not overlap with the phase-matched frequencies and DW amplification disappear. Fig 8 shows the output spectrum evolution as function of pump wavelength for $1 \mathrm{~W}$ input power. In this figure we can see that DW gives a significant contribution to spectral broadening only when pumping just above $\lambda_{\text {ZD }}$. For increasing pumping wavelength, their intensity is decreasing and the spectral gap between the residual pump 
and the DW peak is increasing. For $\lambda_{\mathrm{P}}>1566 \mathrm{~nm}$, there is no DW amplification and the whole SC spectrum lies above $\lambda_{\mathrm{ZD}}$.

(a)

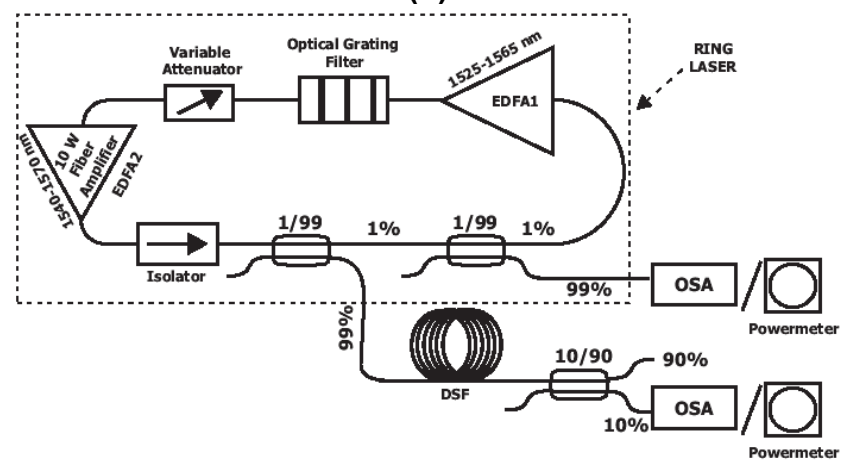

(b)

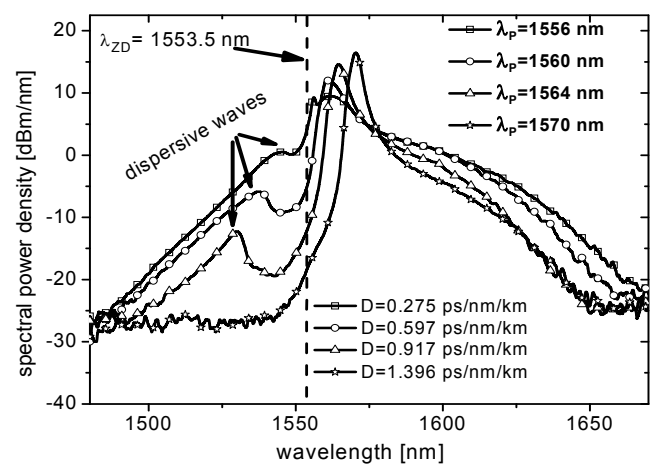

Fig 7. (a) Experimental setup used to study the chromatic dispersion influence in SC-CW broadening. EDFA: erbium-doped fiber amplifier, FDF: flat dispersion fiber, OSA: optical spectrum analyzer. (b) Dispersive wave generation for a input power of $1 \mathrm{~W}$.

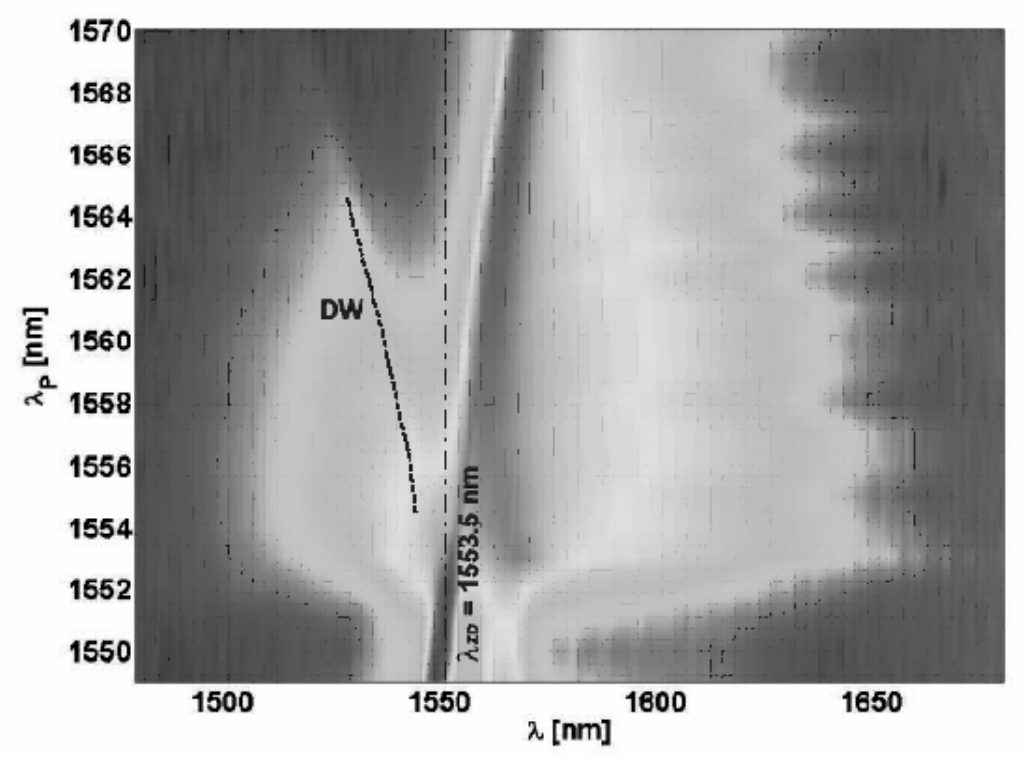

Fig 8. Output spectra as function of pump wavelength and $\lambda_{P}$ (vertical axis) $\left(\mathrm{P}_{\mathrm{IN}}=1 \mathrm{~W}\right)$.

In all the above described experiments the increasing of the pump power normally produces the increased the SC width, but the average power spectral density (APSD) would not grow with pump power and could even be reduced. For many applications, a fixed SC width should be desirable and the control of the power spectra density by simply changing the input pump power. This possibility was demonstrated in our laboratory for CW-SC by using a fiber with two zero dispersion wavelengths (flat dispersion fiber, FDF) ${ }^{22}$, and using the tunable erbium-doped fiber laser shown in Fig 7(a). This FDF has two $\lambda_{\text {ZD }}$ at 1390 and $1652 \mathrm{~nm}$, then the fiber exhibits small anomalous dispersion in the region of $1500 \mathrm{~nm}$ and can be pumped with high-power CW in order to obtain SC. In this case the SC power was bounded between the two $\lambda_{Z D}$ of the flat-dispersion fiber.

Fig 9 shows the spectra measured at the output of the $6 \mathrm{~km}$ FDF pumped at $1550 \mathrm{~nm}$ for different input powers. The spectral bounding of the SC is achieved in this fiber between the pump wavelength at $1550 \mathrm{~nm}$ and the second DW generated at about $1675 \mathrm{~nm}$. This DW as in the before described case is caused by solitons which are shifted just below the second $\lambda_{\mathrm{ZD}}$. The limits of the SC remain constant from $4 \mathrm{~W}$ up to $7 \mathrm{~W}$. The position of the second DW did not 
depend on the pump power. As a consequence, for a fixed pump wavelength it is possible to control the average power spectra density of the SC by simply tuning the pump power. For instance, pumping at $1550 \mathrm{~nm}$, an increase in the input pump power from 4 to $7 \mathrm{~W}$ led to a raise in the APSD of the SC from $0.96 \mathrm{dBm} / \mathrm{nm}$ to $1.87 \mathrm{dBm} / \mathrm{nm}$. Unfortunately, the increase in APSD is not proportional to the pump power, because the losses in the fiber are strongly wavelengthdependent (at longer wavelengths). When the pump power is increased, the longer wavelength part of the SC is being enhanced.

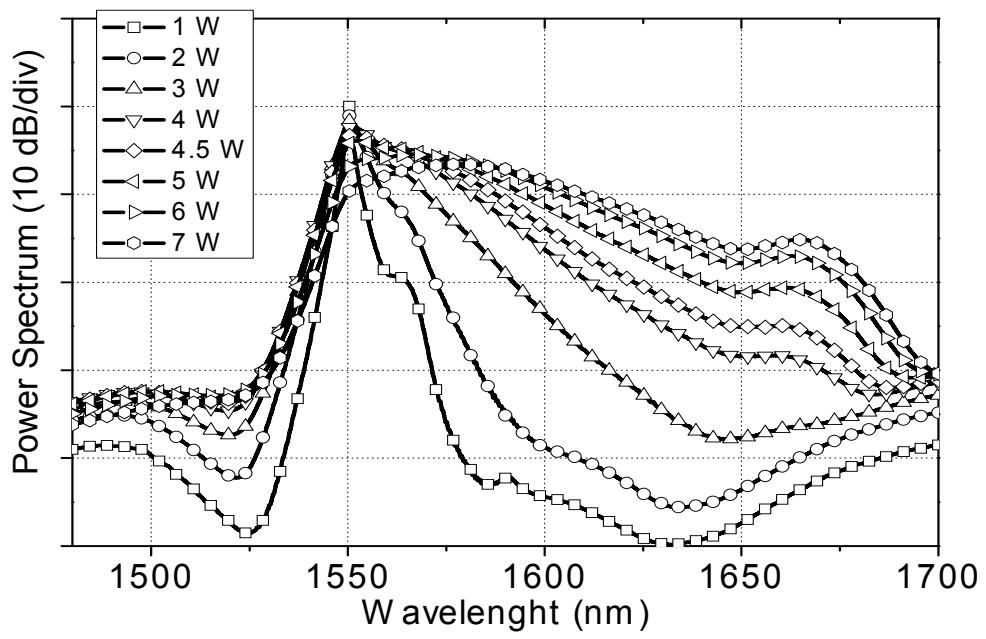

Fig 9. CW-SC obtained in a $6 \mathrm{~km}$ FDF pumped at $1550 \mathrm{~nm}$ wavelength for different input powers.

\section{CONCLUSIONS}

Supercontinuum generation is a process where laser light is converted into a very spectrally broadband light by nonlinear processes. A short review of the physical processes involved in SC generation from continuous wave pump has been performed. To obtain supercontinua from continuous wave pumps, first MI breaks-up the CW pump beam into multiple solitons. These solitons then undergo Raman self-frequency shift, extending the SC on the long wavelength, while simultaneously shedding some energy in the form of blue-shifted dispersive waves. Numerical simulations have demonstrated that the partially-coherent character of the CW pump beam is actually the key ingredient to interpret the smooth broadening achieved under $\mathrm{CW}$ pumping. The incoherence of the pump laser manifests itself by random phase and intensity fluctuations in the input wave, and that makes the dynamics of CW-SC generation much more complex than in the pulsed case. Because of these fluctuations, the MI induced Raman solitons are generated with widely different parameters, so that, on a time scale comparable to the coherence time of the pump, the output light is made up of a random succession of solitons. The SC spectral smoothness simply results from the fact that this distribution of solitons covers the entire SC bandwidth and is naturally averaged by measuring instruments.

A review of some experiments performed in our laboratory for the SC generation in standard communication fibers and using CW lasers as pump source has been presented. The influence of chromatic dispersion on $\mathrm{n}$, by sweeping the input laser wavelength in order to pump under different values of chromatic dispersion but similar values of nonlinear coefficient, attenuation, and higher-order dispersive terms. For a given fiber length, our results show that, at low input powers, chromatic dispersion plays a dominant role on nonlinear pump spectral broadening, giving rise to a broader spectrum when pumping just above the zero-dispersion wavelength of the fiber. When the input power approaches the Raman threshold, however, the width of the generated supercontinuum spectrum is mostly due to the Raman effect, hence more independent of the value of the chromatic dispersion coefficient.

Finally a confined CW-SC generation spectrally bounded by using a fiber with two $\lambda_{\text {ZD }}$ is presented. We achieved a quite flat SC spanning from $1550 \mathrm{~nm}$ to $1700 \mathrm{~nm}$. The APSD in the fiber could be tuned in the range of several $\mathrm{mW} / \mathrm{nm}$. It is worth noting that all the pump power has been converted into the $\mathrm{SC}$, leading to a good figure of merit compared with previously reported CW-SC results. 


\section{ACKNOWLEDGMENTS}

We acknowledge financial support from the Ministerio de Educacion y Ciencia through projects TEC2006-09990-C0201 and TEC2006-09990-C02-02, the support from CSIC through project MeDIOMURO, the support from the Comunidad Autónoma de Madrid through the projects FUTURSEN S-0505/AMB/000374 and FACTOTEM S$505 / \mathrm{ESP} / 000417$.

\section{REFERENCES}

[1] Prabhu, M., Kim, N.S., Ueda, K., "Ultra-broadband CW supercontinuum generation centered at $1483.4 \mathrm{~nm}$ from Brillouin/Raman fiber laser.” Japan. J. Appl. Phys. 39, L291-L293 (2000).

[2] Alfano, R. R. and Shapiro, S. L., "Emission in the region 4000 to $7000 \AA$ via four-photon coupling in glass." Phys. Rev. Lett. 24, 584-587 (1970).

[3] Abeeluck, A. K., Headley, C. and Jørgensen, C. G., "High-power supercontinuum generation in highly nonlinear, dispersionshifted fibers by use of a continuous-wave Raman fiber laser." Opt. Lett. 29, 2163-2165 (2004).

[4] Birks, T. A., Wadsworth, W. J. and Russell, P. St. J., "Supercontinuum generation in tapered fibers," Opt. Lett. 25, 1415-1417 (2000).

[5] Avdokhin, A. V., Popov, S. V. and Taylor, J. R., "Continuous-wave, high-power, Raman continuum generation in holey fibers," Opt. Lett. 28, 1353-1355 (2003).

[6] Kobtsev, S. M. and Smirnov, S.V., "Modelling of high-power supercontinuum generation in highly nonlinear, dispersion shifted fibers at CW pump." Opt. Express 13, 6912-6918 (2005).

[7] Gonzalez-Herraez, M., Martin-Lopez, S., Corredera, P., et al. "Supercontinuum generation using a continuouswave Raman fiber laser", Opt. Commun. 226 (1-6): 323-328, (2003)

[8] L. Abrardi, S. Martin-Lopez, A. Carrasco-Sanz, P. Corredera, ML. Hernanz and M. Gonzalez-Herraez, "Optimized all-fiber supercontinuum source at $1.3 \mu \mathrm{m}$ generated in a stepwise dispersion-decreasing-fiber arrangement", J. Lightwave Technol., 25, 2098-2012, 2007

[9] S. Martin-Lopez, M. Gonzalez-Herraez, P. Corredera P, Hernanz, M. L. and Carrasco, A., "Gain-flattening of fiber Raman amplifiers using nonlinear pump spectral broadening", Opt. Comun. 242 (4-6), 463-469, (2004)

[10] Ellingham, T. J., Ania-Castañón, J. D., Turitsyn, S.K., Pustovskikh, A., Kobtsev, S. and Fedoruk, M. P., "Dualpump Raman amplification with increased flatness using modulation instability", Opt. Express 13 (2005) 10791084.

[11] Smirnov, S. V., Ania-Castanon, J. D., Ellingham, T. J., Kobtsev, S. and Turitsyn, S. K., "Optical spectral broadening and supercontinuum generation in telecom applications." Opt. Fiber Technol., 12, 122-147 (2006).

[12] Fercher, A. F., Drexler, W., Hitzenberger, C. K. and Lasser, T., "Optical coherence tomography-principles and applications." Rep. on Progr. in Phys. 66, 239-303 (2003).

[13] Martin-Lopez, S., Gonzalez-Herraez, M., Carrasco-Sanz, A., Vanholsbeeck, F., Coen, S., Fernandez, H., Solis, J., Corredera, P. and Hernanz, M. L.,. "Broadband spectrally flat and high power density light source for fibre sensing purposes". Meas. Sci. Technol.17, 1014-1019 (2006)

[14] Agrawal, G., [Nonlinear fiber optics], Academic press, (2001)

[15] Dudley, J. M., Genty, G. and Coen, S., "Fundamental limits to few cycle pulse generation from compression of supercontinuum spectra generated in photonic crystal fibers," Opt. Express, 12, 2423-2428, (2006).

[16] Mollenauer, L. F., Stolen, R. H. and Gordon, J.P., "Experimental observation of picosecond pulse narrowing and solitons in optical fibers," Phys. Rev. Lett., vol. 45, no. 13, pp. 1095-1098, 1980.

[17] Mollenauer, L.F., Stolen, R. H., Gordon, J. P. and Tomlinson, W. J., "Extreme picosecond pulse narrowing by means of soliton effect in single-mode optical fibers," Opt. Lett., vol. 8, no. 5, pp. 289-291, 1983.

[18] Martin-Lopez, S., Gonzalez-Herraez, M., Carrasco-Sanz, A., Corredera, P., Abrardi L. and Hernanz, M. L., "Experimental investigation on the effect of pump incoherence on nonlinear pump spectral broadening and continuous-wave supercontinuum generation” Opt. Lett. 31, 3477-3479, (2006)

[19] Vanholsbeeck, F., Martin-Lopez, S., Gonzalez-Herraez, M. and Coen, S., "The role of pump incoherence in continuous-wave supercontinuum generation," Opt. Express 13, 6615- 6625 (2005).

[20] Martin-Lopez, S., Gonzalez-Herraez, M., Corredera, P., Hernanz, M. L. and Mendez, J. A., "Temperature effects on supercontinuum generation using a continuous-wave Raman fiber laser". Optics Communications 267, 193-196 (2006) 
[21] Nicholson, J. W., Abeeluck, A. K., Headley, C., Yan, M. F. and Jørgensen, C. G., "Pulsed and continuous-wave supercontinuum generation in highly nonlinear, dispersion-shifted fibers," Appl. Phys. B B77, 211-218 (2003).

[22] Mussot, A., Lantz, E., Maillotte, H., Sylvestre, T., Finot, C. and Pitois, S., "Spectral broadening of a partially coherent CW laser beam in single-mode optical fibers," Opt. Express 12, 2838-2843 (2004).

[23] Abrardi, L., Martin-Lopez, S., Carrasco-Sanz, A., Corredera, P., Hernanz., M. L. and Gonzalez-Herraez, M., "Experimental Study on the Role of Chromatic Dispersion in Continuous-Wave Supercontinuum Generation", J. Lightwave Technol. 27, 426-435, (2009).

[24] Martin-Lopez, S., Abrardi, L., Corredera, P., Gonzalez-Herraez, M. and Mussot, A., "Spectrally-bounded continuous-wave supercontinuum generation in a fiber with two zero-dispersion wavelengths". Opt. Express, 16, 6745-6755 (2008). 